\title{
The Confined Helium Atom in Hyperspherical Coordinates
}

\author{
M. N. Guimarães \& F. V. Prudente
}

\section{Introduction}

This work aims to develop and implement an efficient theoretical and computational method for the non-relativistic quantum study of spatially confined two electrons atoms. Especially, in recent decades, studies on simply and doubly excited states of helium atom have attracted considerable attention in atomic physics. ${ }^{1}$ Additionally, advances in semiconductor technology have increasingly attracting the interest of physicists and chemists for investigating new quantum objects obtained by the confinement of electrons, atoms or molecules by potential models. $^{2}$

\section{Methods}

Our methodology is based on the variational formalism and hyperspherical coordinates fixed in space, ${ }^{1}$ consisting of one hyperradius, $\rho$, and five hyperangles, $\Omega$, being applied to study the helium atom whose Hamiltonian operator for the time-independent Schrödinger equation in this coordinate system is given by where $\mu$ depends on

$$
H=-\frac{\hbar^{2}}{2 \mu}\left(\frac{\partial^{2}}{\partial \rho^{2}}+\frac{5}{\rho} \frac{\partial}{\partial \rho}\right)+\frac{\hbar^{2}}{2 \mu \rho^{2}} \Lambda^{2}+V
$$

the masses, $\Lambda$ is the hyperangular momentum operator containing all the angular variables ${ }^{3}$ and the confinement potential is given by the expression

$$
V(\rho, \Omega)=\frac{1}{4 \pi \epsilon_{0}} \frac{C(\Omega)}{\rho}+\omega(\rho)
$$

with the first term representing the Coulomb interaction and the second term representing a harmonic isotropic confinement:

$$
V(\rho, \Omega)=\frac{1}{4 \pi \epsilon_{0}} \frac{C(\Omega)}{\rho}+\omega(\rho)
$$

Also we employed the finite element method for expansion of the wave function in terms of a finite set of local basis functions. In particular, we propose a modification in its $p$-version ( $\mathrm{p}$-FEM) assuming that the Hamiltonian is invariant under a reflection around a midpoint, then the parity is preserved. In this case, we propose to use the p-FEM to build, in a simple way, basis functions adapted to the parity of the wave function. In Figure 1 we show, as an example, the eigenfunction of the seventh excited state of one-dimensional harmonic oscillator problem together with the basis functions utilized to expand an even function. Also we utilize the self-consistent finite element method ${ }^{4}$ to optimize the elements mesh, which, among other things, provide a reduction in the matricial dimensions of the problem. 
with the first term representing the Coulomb interaction and the second term representing a harmonic isotropic confinement:

$$
\omega(\rho)=\frac{1}{2} \omega^{2} \rho^{2}
$$

Also we employed the finite element method for expansion of the wave function in terms of a finite set of local basis functions. In particular, we propose a modification in its p-version (p-FEM) assuming that the Hamiltonian is invariant under a reflection around a midpoint, then the parity is preserved. In this case, we propose to use the p-FEM to build, in a simple way, basis functions adapted to the parity of the wave function. In Figure 1 we show, as an example, the eigenfunction of the seventh excited state of one-dimensional harmonic oscillator problem together with the basis functions utilized to expand an even function. Also we utilize the self-consistent finite element method4 to optimize the elements mesh, which, among other things, provide a reduction in the matricial dimensions of the problem.

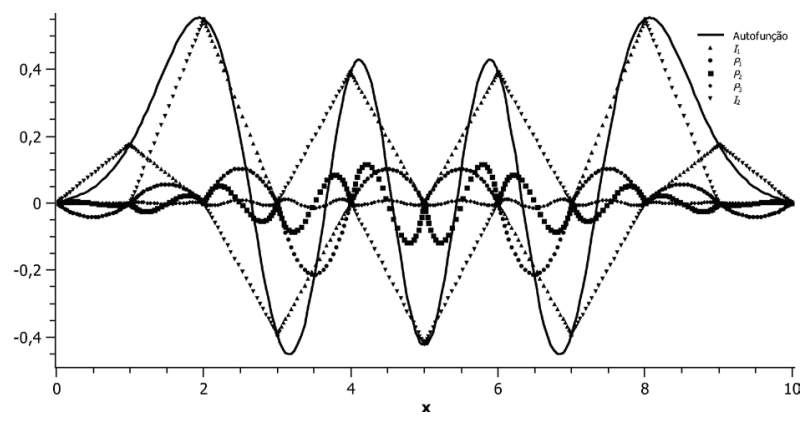

Figure 1. Eigenfunction of seventh excited state of the one-dimensional harmonic oscillator and their symmetry-adapted basis functions using the FEM.

\section{Results and Discussion}

Our emphasis is given to the calculation of the energy levels in atomic units. All calculations were made using a computational implementation in Fortran. Because of the symmetry of the Hamiltonian solutions of the hyperangular equation can be divided into even and odd. Therefore, we can apply the finite element method with basis functions appropriate to symmetry of the problem in which we had argued in the previous section. Examining the singlet states, $\mathrm{S}=0$, of helium atom with total angular momentum $\mathrm{L}=0$ it is possible to note that the conditions given by problem equations determine that only even solutions will exist.

The use of hyperspherical coordinates enables propose an adiabatic separation between the hyperradius and hyperangles in the same sense as the usual adiabatic model for a molecule. In Figure 2 are shown the potential curves for the first eigenvalues obtained from the solution of hyperangular part considering $\omega=0$, corresponding to free helium atom, and $\omega=0.1$ a.u., corresponding to the harmonic confinement. We note that the potential curve of Figure 2 for $\omega=0$ are compatible with the graphics in the literature. 5 On the other hand, in the confinement situation, we note that as the hyperradius increases the angular eigenvalues tend to feel more the influence of the confinement potential increasing rapidly its value as compared to the free situation.

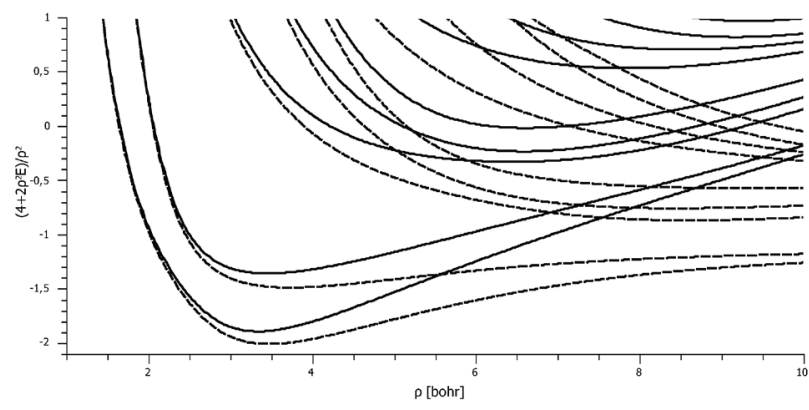

Figure 2. Potential curves of the solution the hyperangular part of the problem of harmonically confined helium atom. Dashed line corresponds to $\omega=0$ a.u. and solid line corresponds $\omega=0.1$ a.u.

In the Table 1 we show some energy values of singlet states with $\mathrm{L}=0$ for two confinement intensities, $\omega$. We note that even in a weak confinement the levels are already significantly different from the free helium atom presented in literature ${ }^{5}$. Although we have no numerical data for comparison we note that our results have values 
consistent with those presented in Figure 6 of the Sako and Diercksen paper6.

Also in Figure 3, we show the energy spectrum, E, depending on the intensity of confinement, $\omega$. In this figure it can clearly be observed split between the levels with $\mathrm{L}=0$ when $\omega$ increases in value which becomes more evident as the confinement is getting stronger. Indeed, for large values of $\omega$, the influence of Coulomb repulsion on the correlation of electrons is smaller and its movements become mostly governed by the harmonic potential of confinement that affects the state with higher energies.

Table 1. Energy levels of helium atom, with $\mathrm{L}=0$, confined by a harmonic isotropic potential obtained by p-FEM.

\begin{tabular}{|c|c|c|}
\hline State & \multicolumn{2}{|c|}{ Energy [a.u.] } \\
\hline & $\omega=0.1$ & $\omega=0.5$ \\
\hline $1 \mathrm{~S}$ & -2.892146 & -2.655916 \\
\hline $2 \mathrm{~S}$ & -2.048908 & -1.006816 \\
\hline $3 \mathrm{~S}$ & -1.756004 & 0.115915 \\
\hline
\end{tabular}

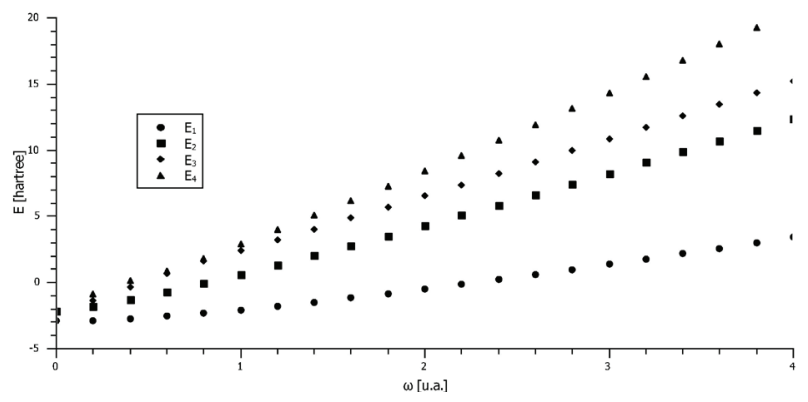

Figure 3. Energy spectrum, E, depending on the intensity of the confinement, $\omega$, for the helium atom confined by an isotropic harmonic potential. computer codes based on new variational methods for solving the time-independent Schrödinger equation which are capable of performing extensive calculations of spatially confined atomic systems.

\section{Acknowledgments}

The authors are grateful for the support given from the CNPq.

\section{References}

1. C. D. Lin, Phys. Rep., 257, 1, 1995.

2. W. Jaskólski, Phys. Rep., 271, 1, 1996.

3. F. T. Smith., Phys. Rev., 120, 1058, 1960.

4. E. M. Nascimento, F. V. Prudente, M. N. Guimarães and A. M. Maniero, J. Phys. B: At. Mol. Opt. Phys, 44, 015003, 2011.

5. J. J. De Groote, M. Masili and J. E. Hornos, J. Phys. B: At. Mol. Opt. Phys., 31, 4755, 1998.

6. T. Sako and H. F. Diercksen, J. Phys. B.: At. Mol. Opt. Phys., 36, 1681, 2003.

\section{N. Guimarães* \& F. V. Prudente}

Instituto de Física. Universidade Federal da Bahia. Salvador, BA.

*mng@ufba.br

\section{Conclusions}

We conclude that the procedure focused on p-FEM was overall quite efficient. We believe that the realization of this work represents a starting point to achieving our primary goal in this research field: the building of 